\title{
Timing of frozen-thawed embryo transfer after controlled ovarian stimulation in a non-elective freeze-all policy
}

\author{
Jialyu Huang", Xuefeng Lu\#, Qin Xie, Jiaying Lin, Renfei Cai, Yanping Kuang \\ Department of Assisted Reproduction, Shanghai Ninth People's Hospital, Shanghai Jiao Tong University School of Medicine, Shanghai 200011, \\ China \\ Contributions: (I) Conception and design: J Huang, X Lu, R Cai, Y Kuang; (II) Administrative support: J Huang, X Lu, R Cai; (III) Provision of study \\ materials or patients: Q Xie, J Lin, R Cai, Y Kuang; (IV) Collection and assembly of data: J Huang, X Lu; (V) Data analysis and interpretation: J \\ Huang, X Lu, Y Kuang; (VI) Manuscript writing: All authors; (VII) Final approval of manuscript: All authors. \\ "These authors contributed equally to this work. \\ Correspondence to: Renfei Cai; Yanping Kuang. Department of Assisted Reproduction, Shanghai Ninth People's Hospital, Shanghai Jiao Tong \\ University School of Medicine, 639 Zhizaoju Rd., Shanghai 200011, China. Email: cairf1550@126.com; kuangyp9hospital@126.com.
}

\begin{abstract}
Background: Non-elective freeze-all policy has been increasingly utilized in assisted reproductive treatment, but the optimal timing of frozen-thawed embryo transfer (FET) after controlled ovarian stimulation (COS) remains to be investigated.

Methods: This retrospective cohort study included 2,998 patients who underwent their first FETs after the first COS cycles using the non-elective freeze-all strategy from Jan 2013 to Dec 2016 at a tertiary-care academic medical center. Patients were divided into the "immediate" group in which FET took place within the first menstrual cycle after oocyte retrieval, and the "delayed" group where FET started after one or more menstrual cycles following COS.
\end{abstract}

Results: The mean interval between oocyte retrieval and FET was $33.3 \pm 5.8$ days in the immediate group $(\mathrm{n}=280 ; 9.3 \%)$ and $91.3 \pm 19.4$ days in the delayed group $(\mathrm{n}=2,718 ; 90.7 \%)$. Cycles with delayed FET had a significantly lower live birth rate than those with immediate FET before [1,246/2,718 (45.8\%) vs. 156/280 (55.7\%); $\mathrm{P}=0.002]$ and after propensity score matching (PSM) [123/280 (43.9\%) vs. 156/280 (55.7\%); $\mathrm{P}=0.005]$. When controlling for a number of confounding factors by multivariable logistic regression analysis, the risk remained significant with the adjusted odds ratio (aOR) [95\% confidence interval (CI)] of $0.69(0.53-0.90)$ and $0.60(0.42-0.85)$ before and after matching, respectively.

Conclusions: Performing FET immediately within the first menstrual cycle following COS was associated with a higher chance to achieve live birth compared with delaying FET to subsequent cycles in a non-elective freeze-all policy. However, further randomized controlled trials are still needed to confirm this conclusion.

Keywords: Frozen-thawed embryo transfer (FET); controlled ovarian stimulation (COS); freeze-all; live birth

Submitted Aug 08, 2019. Accepted for publication Oct 22, 2019.

doi: $10.21037 /$ atm.2019.11.74

View this article at: http://dx.doi.org/10.21037/atm.2019.11.74

\section{Introduction}

Controlled ovarian stimulation (COS) is a key determinant of in vitro fertilization/intracytoplasmic sperm injection (IVF/ICSI) success, in which multiple follicles develop simultaneously and plenty of oocytes are retrieved within one cycle. Unfortunately, the supraphysiologic hormonal concentrations during this process could also lead to reduced endometrial and subendometrial blood flow (1), advanced maturation of the endometrium (2), altered endometrial immune environment (3) as well as disrupted gene expression profile in relation to endometrial receptivity (4). These detrimental effects consequently translate into the less favorable outcomes of embryo implantation and 
pregnancy rates in fresh transfer cycles $(5,6)$.

In an effort to overcome this problem, cryopreservation of all embryos after COS for subsequent thawing and transfer, namely the freeze-all policy, has been increasingly utilized in the past decade (7). Compared with fresh embryo transfer, this strategy demonstrates its great benefits in decreasing the risk of ovarian hyperstimulation syndrome (OHSS) while improving pregnancy, obstetrical and neonatal outcomes (8-12). To further minimize the potential carryover effects of COS on endometrial and ovarian function, a majority of physicians often opt to delay the frozen-thawed embryo transfer (FET) for at least one menstrual cycle, as evidenced by the protocol of recent clinical trials (9-11) as well as a web-based survey from IVFWorldwide (13).

Although the widespread preference of FET deferral after COS may be based on the best of intentions, this approach is actually empirical with limited scientific evidence $(14,15)$. Instead, delaying FET may prolong the time to achieve pregnancy and cause unnecessary distress and anxiety to infertile couples, which could lower the chance of pregnancy and increase the incidence of treatment discontinuation (16-18). Thus far, only a handful of studies (19-22) have assessed the optimal timing of FET following COS in a freeze-all protocol with conflicting results, highlighting the lack of both understanding and agreement on this matter. In addition, these studies are hampered by relatively small sample size, selection bias from elective freeze-all strategy and incomplete adjustment of potential confounding factors. Given the medical and financial significance of the issue, we consider these preliminary results deserve further scrutiny and clarification.

The objective of the present study was to investigate if performing FET immediately within the first menstrual cycle after COS or delaying FET to subsequent cycles has any impact on reproductive outcomes in the non-elective freeze-all policy.

\section{Methods}

\section{Study design and participants}

This was a retrospective cohort study conducted at the Department of Assisted Reproduction of Shanghai Ninth People's Hospital affiliated with Shanghai Jiao Tong University School of Medicine. The study protocol was approved by the hospital's Ethics Committee (Institutional Review Board). Infertile women who underwent their first FETs after the first IVF/ICSI cycles using the freezeall policy were enrolled from January 2013 to December 2016. To minimize potential bias, we excluded patients who met one of the following criteria: (I) abnormal results on parental karyotyping; (II) documented history of unilateral oophorectomy or recurrent spontaneous abortion, defined as three or more spontaneous pregnancy losses; (III) previous diagnosis of congenital (e.g., septate uterus and duplex uterus) or acquired (e.g., submucosal myoma and adenomyosis) uterine anomalies; (IV) use of donor sperm or sperm from percutaneous testicular and epididymal aspiration; (V) moderate and severe OHSS during COS; (VI) embryo cryopreservation for over 120 days $(14,15)$; (VII) core information missing in the electronic medical records (e.g., endometrial thickness). None of the patients at our center underwent cycles with donated oocytes, in vitro maturation or preimplantation genetic diagnosis.

\section{Ovarian stimulation and embryo culture}

All patients underwent COS with exogenous gonadotrophins by using progestin-primed ovarian stimulation (PPOS) or gonadotropin-releasing hormone agonist (GnRH-a) short protocol (23). Briefly, patients in the GnRH-a short protocol were injected daily with $0.1 \mathrm{mg}$ triptorelin (Decapeptyl, Ferring Pharmaceuticals, Germany) from menstrual cycle day 2 (MC2) onward and 150-225 IU human menopausal gonadotropin (hMG; Anhui Fengyuan Pharmaceutical Co., China) from MC3 onward until trigger. In the PPOS regimen, patients were administered daily with $10 \mathrm{mg}$ medroxyprogesterone acetate (Shanghai Xinyi Pharmaceutical Co., China) and 150-225 IU hMG from MC3 to the day of triggering. The doses of hMG were adjusted according to ovarian response, as assessed by transvaginal ultrasound (TVU) examination and serum estradiol $\left(\mathrm{E}_{2}\right)$ concentration. When at least three follicles reached $18 \mathrm{~mm}$ in diameter or one dominant follicle reached $20 \mathrm{~mm}$, final oocyte maturation was triggered with 5,00010,000 IU human chorionic gonadotropin (hCG; Lizhu Pharmaceutical Trading Co., China) for the GnRH-a short protocol, while the single use of hCG $(5,000-10,000 \mathrm{IU})$ or triptorelin $(0.1-0.2 \mathrm{mg})$, or dual trigger by a low dose of hCG (1,000 IU) and triptorelin $(0.1 \mathrm{mg})$ was applied for the PPOS protocol $(23,24)$.

Oocyte retrieval was scheduled at 34-36 h after trigger. The aspirated oocytes were fertilized by conventional IVF and/or ICSI according to semen parameters. The zygotes were transferred and cultured in the Continuous Single 
Culture (Irvine Scientific, USA) throughout the entire developmental stage. Embryos were graded according to the Cummins's criteria (25) on day 3 after oocyte retrieval and top-quality embryos (grade I and II) were selected for vitrification. Suboptimal embryos (grade III and IV) were subjected to extended culture and morphologically good blastocysts (grade $\geq 3 \mathrm{BC}$ ) based on the Gardner and Schoolcraft scoring system (26) were selected for vitrification on day 5 or 6 . The vitrification and thawing procedures were performed the same as those presented previously (23).

\section{Endometrial preparation and embryo transfer}

The timing of FET was defined as the interval between oocyte retrieval and the start of the first FET cycle. Patients were divided into the "immediate" group in which the FET took place within the first menstrual cycle after oocyte retrieval, and the "delayed" group where the FET started after one or more menstrual cycles following COS.

Modified natural cycles were recommended for patients with regular menstrual cycles. Follicular monitoring was performed using TVU and blood sampling every 2 days from MC10 onward. Once the dominant follicle was $\geq 17 \mathrm{~mm}$ in diameter with $\mathrm{E}_{2}>150 \mathrm{pg} / \mathrm{mL}$ and progesterone (P) $<1 \mathrm{ng} / \mathrm{mL}$, a bolus of urinary hCG $(5,000 \mathrm{IU})$ was administered for ovulation trigger. The injection was scheduled in the same afternoon in cases of luteinizing hormone ( $\mathrm{LH})$ surge ( $\mathrm{LH} \geq 20 \mathrm{IU} / \mathrm{L})$, or at night (9:00 $\mathrm{pm})$ if $\mathrm{LH}<20 \mathrm{IU} / \mathrm{L}$. Respectively, exogeneous $\mathrm{P}$ supplementation (dydrogesterone $40 \mathrm{mg} / \mathrm{d}$; Duphaston, Abbott Biologicals, USA) was commenced 2 and 3 days later, followed by cleavage-stage embryo transfer 4 and 5 days later or blastocyst transfer 6 and 7 days later. For patients with irregular menstrual cycles, artificial cycles were induced with sequential provision of oral $\mathrm{E}_{2}(8 \mathrm{mg} / \mathrm{d}$; Fematon-red tablets, Abbott Biologicals, USA) and P both orally (dydrogesterone $40 \mathrm{mg} / \mathrm{d}$; Fematon-yellow tablets, Abbott Biologicals, USA) and vaginally $(400 \mathrm{mg} / \mathrm{d}$; Utrogestan, Besins Manufacturing, Belgium). The standard length of $E_{2}$ supplementation was 14 days but could be extended for a maximum of 7 days if the endometrial thickness was below $7 \mathrm{~mm}$. The time of embryo thawing and transfer was set on the 3 rd or the 5 th day after $\mathrm{P}$ administration depending on the embryo stage. Up to two embryos per patient were transferred in each FET cycle. When a pregnancy was achieved, luteal phase support was continued to 10 weeks of gestation.

\section{Outcome measures}

The primary outcome of the study was the live birth rate per cycle. Secondary outcomes included the rates of biochemical pregnancy, implantation, clinical pregnancy, early miscarriage and ongoing pregnancy.

Live birth was defined as the delivery of a viable infant at 24 weeks or more of gestation. Biochemical pregnancy was defined as a serum $\beta$-hCG level $\geq 5 \mathrm{IU} / \mathrm{L}$ at 14 days after FET. The implantation rate was calculated as the number of gestational sacs visualized on TVU divided by the number of embryos transferred. Clinical pregnancy was identified as the presence of at least one gestational sac with or without fetal heart activity at 7 gestational weeks. Early miscarriage was defined as spontaneous pregnancy termination prior to the gestational age of 12 weeks. Ongoing pregnancy was defined as a viable pregnancy beyond 12 weeks' gestation.

\section{Statistical analysis}

Statistical analysis was performed with the Statistical Package for the Social Sciences (version 20.0; SPSS Inc., USA) and R statistical programming language (version 3.6.0; R Foundation for Statistical Computing, Austria). The normality of continuous variables was tested by the ShapiroWilk test as well as visual inspection of histograms and Q-Q plots. Data were presented as mean with standard deviation and differences between groups were compared by Student's $t$-test or Mann-Whitney $\mathrm{U}$ test. For categorical variables, data were described as frequency with rate and compared by Chi-square test or Fisher's exact test as appropriate.

To balance baseline characteristics between the two groups, a one-to-one propensity score matching (PSM) model was established using the nearest-neighbor matching algorithm. The variables chosen for matching included all potential confounders, namely maternal age (continuous), maternal body mass index (continuous), gravidity ( 0 or $\geq 1$ ), parity ( 0 or $\geq 1)$, infertility duration (continuous), infertility diagnosis (tubal factor, male factor, other, unexplained or combined), ovarian stimulation protocol (GnRH-a short or PPOS), duration of stimulation (continuous), total hMG dose (continuous), type of triggering (hCG, GnRH-a or dual trigger), peak $\mathrm{E}_{2}$ level (continuous), number of oocytes retrieved (continuous), fertilization method (IVF, ICSI or IVF + ICSI), FET endometrial preparation (modified natural cycle or artificial cycle), endometrial thickness (continuous), number of embryos transferred (single or double) and embryo stage at transfer (cleavage 
or blastocyst). The balance between the two groups after matching was evaluated by the standardized mean difference $(<0.1)$.

The association between FET timing after COS and pregnancy outcomes was evaluated before and after PSM by both univariable and multivariable logistic regression analysis. All the aforementioned risk factors for matching were introduced into the regression equation for adjustment by the enter method, except the number of retrieved oocytes due to its collinearity with peak $\mathrm{E}_{2}$ level according to the variance inflation factor. Specially, to better distinguish the degree of ovarian response, peak $\mathrm{E}_{2}$ level during COS was categorized for adjustment in regular increments of $1,000 \mathrm{pg} / \mathrm{mL}:<1,000,1,000-1,999,2,000-2,999,3,000$ $3,999,4,000-4,999$ and $\geq 5,000 \mathrm{pg} / \mathrm{mL}$.

All $\mathrm{P}$ values were two-sided, and $\mathrm{P}<0.05$ was considered to be statistically significant.

\section{Results}

A total of 2,998 first FET cycles were included in the analysis. There were $280(9.3 \%)$ patients who started FET within the first menstrual cycle after COS, while the majority of FET cycles $(n=2,718 ; 90.7 \%)$ were initiated after one or more menstrual cycles.

Table 1 presented the baseline demographics and cycle characteristics before (left column) and after (right column) PSM. Before individual matching, the two groups differed significantly in the proportion of nulligravida, infertility diagnosis, total hMG dose, type of triggering, peak $\mathrm{E}_{2}$ level, endometrial preparation and endometrial thickness. The mean interval between oocyte retrieval and FET was $33.3 \pm 5.8$ days in the immediate group and $91.3 \pm 19.4$ days in the delayed group $(\mathrm{P}<0.001)$. After PSM, all baseline characteristics of 280 patients in each group were similarly adjusted. The distributions of propensity score and standardized difference were shown in Figure 1.

Pregnancy outcomes grouped by FET timing were shown in Table 2. We found significantly reduced rates of implantation ( $37.8 \%$ vs. $44.2 \%$; $\mathrm{P}=0.004)$, clinical pregnancy (54.3\% vs. $62.9 \%$; $\mathrm{P}=0.006$ ), ongoing pregnancy ( $48.1 \%$ vs. $58.2 \% ; \mathrm{P}=0.001)$ and live birth $(45.8 \%$ vs. $55.7 \% ; \mathrm{P}=0.002)$ in the delayed FET group compared with the immediate FET group before matching. The risks were maintained after controlling for a number of confounding factors, with the adjusted odds ratio (aOR) of 0.73 [95\% confidence interval (CI), 0.56-0.95], 0.68 (95\% CI, 0.52-0.88) and 0.69 (95\% CI, 0.53-0.90) for clinical pregnancy, ongoing pregnancy and live birth, respectively (Table 3). Early miscarriage rate did not differ significantly between the two groups in crude analysis (9.3\% vs. $5.1 \% ; \mathrm{P}=0.062)$, but was slightly higher in the delayed FET group after adjustment (aOR 2.15; 95\% CI, 1.05-4.39).

Consistently, re-analysis after PSM showed that delayed FET after COS was associated with significantly decreased chances of clinical pregnancy (aOR 0.66 ; $95 \%$ CI, $0.46-$ 0.94), ongoing pregnancy (aOR 0.59; 95\% CI, 0.42-0.84) and live birth (aOR 0.60; 95\% CI, 0.42-0.85). Furthermore, delayed FET conferred a higher risk of early miscarriage (aOR 2.91; 95\% CI, 1.05-8.06) than immediate transfer, whereas the association with biochemical pregnancy was insignificant before and after adjustment (Table 3).

As demonstrated in Table S1, other common influencing factors of live birth before and after matching included maternal age, parity number, unexplained infertility and number of embryos transferred. Peak $\mathrm{E}_{2}$ level during COS did not reveal significant associations with live birth in subsequent FET cycles.

\section{Discussion}

The results of this retrospective cohort study suggested that performing FET immediately within the first menstrual cycle after oocyte retrieval was associated with a higher chance to achieve live birth compared with delaying FET after one or more menstrual cycles in the freeze-all policy.

The prioritization of time efficiency is not new in the setting of a failed fresh embryo transfer cycle (14,27-29). In 2008, Maas et al. (28) first reported that cycles with delayed FET had a significantly lower clinical pregnancy rate than those with immediate FET after a failed fresh cycle. This study, however, was contradicted later by two retrospective analyses showing that intentional postponement of FET did not enhance or deteriorate pregnancy outcomes $(27,29)$. To add further to the confusion, Volodarsky-Perel et al. (14) found a significantly positive impact of delaying FET on implantation, clinical pregnancy and live birth after failed fresh embryo transfer cycles using the GnRH-a long protocol.

In coincidence with the constant rise in segmented IVF cycles, more recent studies have tried to investigate the optimal timing to perform FET in the freeze-all strategy $(15,19-22,30)$. In the combined analysis of FET cycles following freeze-all and failed fresh embryo transfer, Kaye et al. (15) demonstrated a marginal significance of ongoing pregnancy in favor of delayed FET. On the 
Table 1 Baseline demographics and cycle characteristics before and after propensity score matching



Table 1 (continued) 
Table 1 (continued)

\begin{tabular}{|c|c|c|c|c|c|}
\hline Variables & Immediate $(n=280)$ & \multicolumn{2}{|c|}{ Before matching } & \multicolumn{2}{|c|}{ After matching } \\
\hline No. of embryos transferred, $\mathrm{n}(\%)$ & & & 0.349 & & 0.700 \\
\hline Single & $33(11.8)$ & $272(10.0)$ & & $36(12.9)$ & \\
\hline Double & 247 (88.2) & $2,446(90.0)$ & & $244(87.1)$ & \\
\hline Cleavage stage & $265(94.6)$ & $2,538(93.4)$ & & $262(93.6)$ & \\
\hline Blastocyst stage & $15(5.4)$ & $180(6.6)$ & & $18(6.4)$ & \\
\hline
\end{tabular}

Values are presented as mean \pm SD or number (percentage). BMI, body mass index; COS, controlled ovarian stimulation; GnRH, gonadotropin-releasing hormone; hMG, human menopausal gonadotropin; hCG, human chorionic gonadotropin; IVF, in vitro fertilization; ICSI, intracytoplasmic sperm injection; FET, frozen-thawed embryo transfer; OPU, ovum pick-up.

A
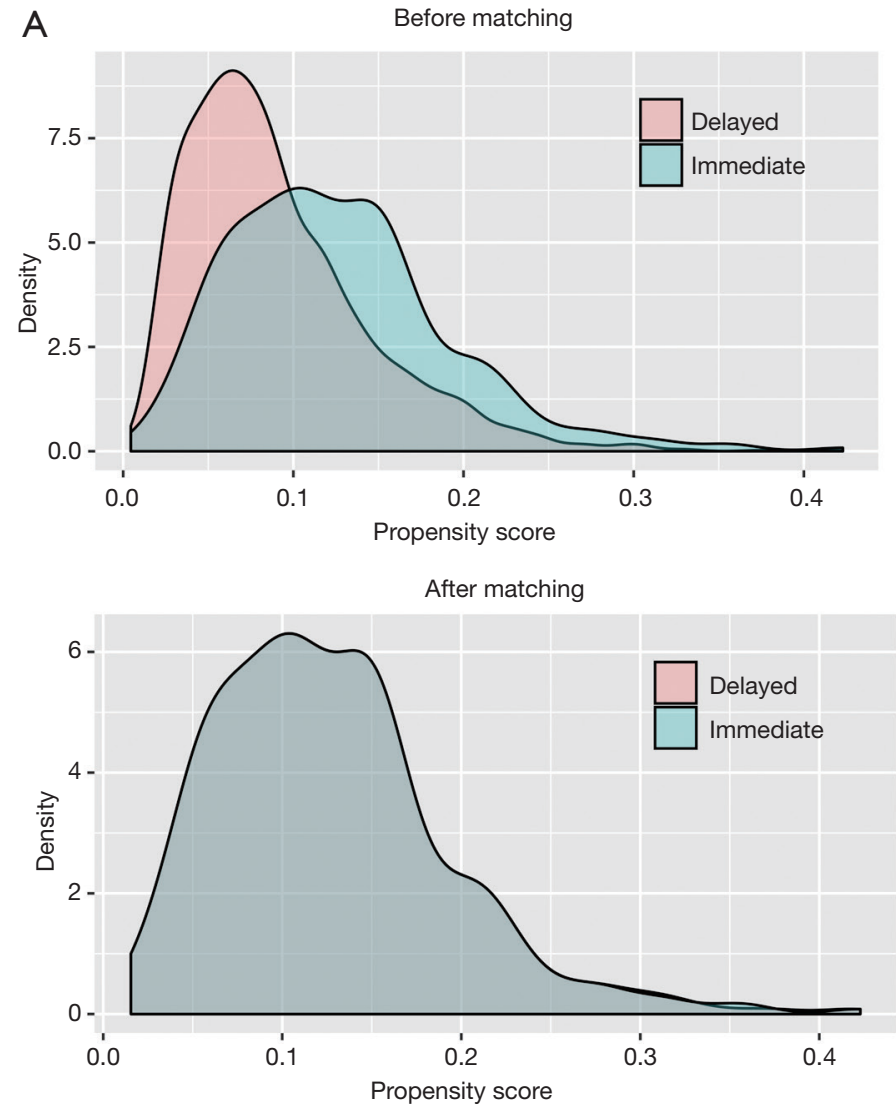

B



Figure 1 Distribution of (A) propensity score and (B) standardized difference before and after matching for delayed and immediate groups.

contrary, a recent study based on a multi-site private IVF clinic database showed that patients with a 25-35 days gap between embryo cryopreservation and transfer had a significantly higher live birth rate than those with a 50
70 days gap (30). In terms of freeze-all cycles alone, SantosRibeiro et al. (19) found a borderline significance of clinical pregnancy in support of immediate FET by reviewing 333 FET cycles from two reproductive centers, while the studies 
Table 2 Pregnancy outcomes grouped by FET timing before and after propensity score matching

\begin{tabular}{|c|c|c|c|c|c|}
\hline Outcomes & $\begin{array}{l}\text { Immediate } \\
\qquad(\mathrm{n}=280)\end{array}$ & \multicolumn{2}{|c|}{ Before matching } & \multicolumn{2}{|c|}{ After matching } \\
\hline Biochemical pregnancy rate, $\mathrm{n}(\%)$ & $183(65.4)$ & $1,624(59.7)$ & 0.068 & $162(57.9)$ & 0.068 \\
\hline Implantation rate, n/N (\%) & 233/527 (44.2) & $1,951 / 5,164(37.8)$ & 0.004 & 199/524 (38.0) & 0.040 \\
\hline Clinical pregnancy rate, $n(\%)$ & $176(62.9)$ & $1,476(54.3)$ & 0.006 & $150(53.6)$ & 0.026 \\
\hline Ongoing pregnancy rate, $\mathrm{n}(\%)$ & $163(58.2)$ & $1,308(48.1)$ & 0.001 & $130(46.4)$ & 0.005 \\
\hline Live birth rate, $\mathrm{n}(\%)$ & $156(55.7)$ & $1,246(45.8)$ & 0.002 & $123(43.9)$ & 0.005 \\
\hline
\end{tabular}

Values are presented as number (percentage). FET, frozen-thawed embryo transfer.

Table 3 Crude and adjusted analysis of pregnancy outcomes following delayed FET versus immediate FET

\begin{tabular}{|c|c|c|c|c|}
\hline Outcomes & \multicolumn{2}{|c|}{ Before matching } & \multicolumn{2}{|c|}{ After matching } \\
\hline Biochemical pregnancy & $0.79(0.61-1.02)$ & $0.82(0.62-1.07)$ & $0.73(0.52-1.02)$ & $0.72(0.50-1.03)$ \\
\hline Clinical pregnancy & $0.70(0.55-0.91)$ & $0.73(0.56-0.95)$ & $0.68(0.49-0.96)$ & $0.66(0.46-0.94)$ \\
\hline Early miscarriage & $1.91(0.96-3.83)$ & 2.15 (1.05-4.39) & 2.37 (1.02-5.49) & $2.91(1.05-8.06)$ \\
\hline Live birth & 0.67 (0.53-0.86) & 0.69 (0.53-0.90) & $0.62(0.45-0.87)$ & $0.60(0.42-0.85)$ \\
\hline
\end{tabular}

OR, odds ratio; FET, frozen-thawed embryo transfer; $\mathrm{Cl}$, confidence interval.

by Lattes et al. (20) and Bourdon et al. (22) demonstrated no significant effects of FET timing on live birth rate after multivariable adjustment.

In contrast to the evidence from aforementioned studies on elective freeze-all programs, only one study analyzed the freeze-all cycles in a non-elective setting and observed no association between FET timing and pregnancy outcomes (21). This work by Ozgur et al. (21) was strengthened by the large sample size of 1,121 cycles, but was limited in generalizability due to its inclusion of only ICSI, artificial and blastocyst transfer cycles. More importantly, patients in the immediate FET group were administrated with GnRH-a for programming of endometrial preparation, while those using oral contraceptive pills for downregulation needed to wait for at least one menstrual cycle and were therefore categorized into the delayed FET group. In this regard, a direct comparison between these two groups was inappropriate and may be greatly complicated by sources of bias.

The finding of our study was in agreement with the studies by Higgins et al. (30) and Santos-Ribeiro et al. (19), and contradicted the other four studies (15,20-22). However, the underlying mechanism for the association between FET timing and pregnancy outcomes has yet to be fully elucidated. While it is hypothesized that delaying FET would be beneficial by allowing the hypothalamicpituitary-ovarian (HPO) axis, dysfunctional corpora lutea and the endometrium to return to their pre-stimulation functionality, accumulating studies have produced results in denial $(14,15,19-22,27,29,30)$. Firstly, a slight delay in ovulation day of about 2 days was indeed encountered in the subsequent natural menstrual cycle following COS, suggesting a gradual resumption of the function of HPO axis (27). Nevertheless, this subtle cycle dynamic did not appear to affect the rates of clinical pregnancy and live birth in immediate FET cycles (27). Secondly, the major concern about corpora lutea dysfunction referred to the abrupt luteolysis, which occurred frequently after GnRH-a triggering without luteal support (31). This assumption, however, was invalid as a growing body of evidence has showed that type of ovulation triggering for COS was not related to pregnancy outcomes in FET cycles $(15,20,32)$. 
Finally, with regard to the endometrium, the present study suggested no effects of peak $\mathrm{E}_{2}$ level during ovarian stimulation on live birth rate in subsequent FET cycles, while another study failed to detect the impact of late follicular progesterone rise (33), both of which implied that the impaired endometrial receptivity could be recovered after the first withdrawal bleeding following oocyte retrieval.

On the contrary, undergoing assisted reproductive treatment is an emotional process for infertile couples who have already shouldered tremendous psychological and physical burden and desire to conceive as soon as possible. In this context, the deferral of FET after COS not only prolongs their time-to-pregnancy, but may also add to unnecessary anxiety and distress, thus posing adverse influences on both pregnancy outcomes and treatment continuation (16-18). On the other hand, the presence of many corpora lutea following COS could lead to high circulating levels of vasodilatory and angiogenic ovarian hormones such as relaxin (34). Due to the association between endometrial angiogenesis and recurrent implantation failure as well as pregnancy loss (35), these corpus luteal products may improve pregnancy outcomes by promoting endometrial growth and development in immediate FET cycles after oocyte retrieval. However, further investigations are warranted to examine these explanations with stronger and more direct evidence.

To the best of our knowledge, the present study was the largest of cohort size in the non-elective freeze-all policy, which minimized the selection bias of patients at high risks of adverse prognosis as in elective cycles (21). Patients were meticulously screened with strict inclusion criteria to allow for a more precise evaluation of the impact of FET timing on reproductive outcomes. Specifically, analysis was limited to the first FET cycles after patients' first IVF/ICSI cycles using the freeze-all strategy, thus eliminating the potential confounders from repeated COS and recurrent FET failures. Furthermore, based on the multivariable regression analysis and the application of PSM, the robustness of the results was tested and reinforced after properly controlling for a wide range of clinically relevant confounding factors. Finally, all these cycle data were derived from a single reproductive center, where laboratory conditions remained unaltered and practice consistency could be guaranteed during the study period.

A major weakness of the study relies on its retrospective design and the possibility of unmeasured confounders, despite the small risk of recall bias because of our prospective data gathering and documentation in a standardized manner. Lack of randomization resulted in the uneven distribution of cases between groups, affected the comparability of baseline variables and may also cause a general loss of statistical power. For example, with a power of 0.8 and type I error of $0.05,389$ patients would be required for each study arm to detect a $10 \%$ difference in live birth rate. The selection bias was minimized as patients at our center are generally suggested to delay FET and the decision to perform immediate FET was made entirely by patients without consideration of COS cycle characteristics. However, the reasons for different deferral number of menstrual cycles were not recorded in our electronic database and may thus lead to bias of results. For instance, patients with residual corpus luteal cysts at MC3 or suboptimal endometrial development may be advised to cancel the FET cycle and wait for the next one. We were also unable to analyze of the day their nonmedical considerations such as cost burden and treatment compliance, as well as psychological states including self-motivation, anxiety level and depression degree. Furthermore, our results were limited to the evaluation of the association between FET timing and pregnancy, and should not be extrapolated to obstetrical (e.g., preeclampsia) and neonatal (e.g., preterm birth) outcomes. In this regard, future trials are of vital importance to assess whether the process of COS has any carryover effect on outcomes beyond pregnancy.

\section{Conclusions}

In summary, our study provided the first-time evidence that immediate FET within the first menstrual cycle following COS was associated with better pregnancy outcomes compared with delayed FET after one or more menstrual cycles in the non-elective freeze-all policy. Therefore, in order to optimize live birth rates while minimizing unnecessary prolongation of time-to-pregnancy, patients should proceed with FET at their earliest convenience after COS. This novel finding not only provides informed evidence to clinicians in medical practice regarding the timing of treatment, but should also be taken into consideration in future clinical research design and implementation.

\section{Acknowledgments}

The authors would like to express sincere gratitude to 
Dr. Sicheng Wu, Clinical Research Center of Shanghai Ninth People's Hospital affiliated to Shanghai Jiao Tong University School of Medicine, for his support in data management and statistical analysis.

Funding: This work was supported by the National Key Research and Development Program of China (Grant No. SQ2018YFC100163) and National Natural Science Foundation of China (Grant No. 81571397, 81771533).

\section{Footnote}

Conflicts of Interest: The authors have no conflicts of interest to declare.

Ethical Statement: The authors are accountable for all aspects of the work in ensuring that questions related to the accuracy or integrity of any part of the work are appropriately investigated and resolved. This study was approved by the Ethics Committee (Institutional Review Board) of Shanghai Ninth People's Hospital affiliated with Shanghai Jiao Tong University School of Medicine (No. 2017-017). Due to the retrospective nature, informed consent was not required, and patients' data were used anonymously.

\section{References}

1. Ng EH, Chan CC, Tang OS, et al. Comparison of endometrial and subendometrial blood flow measured by three-dimensional power Doppler ultrasound between stimulated and natural cycles in the same patients. Hum Reprod 2004;19:2385-90.

2. Kolibianakis E, Bourgain C, Albano C, et al. Effect of ovarian stimulation with recombinant follicle-stimulating hormone, gonadotropin releasing hormone antagonists, and human chorionic gonadotropin on endometrial maturation on the day of oocyte pick-up. Fertil Steril 2002;78:1025-9.

3. Junovich G, Mayer Y, Azpiroz A, et al. Ovarian stimulation affects the levels of regulatory endometrial NK cells and angiogenic cytokine VEGF. Am J Reprod Immunol 2011;65:146-53.

4. Haouzi D, Assou S, Mahmoud K, et al. Gene expression profile of human endometrial receptivity: comparison between natural and stimulated cycles for the same patients. Hum Reprod 2009;24:1436-45.

5. Arslan M, Bocca S, Arslan EO, et al. Cumulative exposure to high estradiol levels during the follicular phase of IVF cycles negatively affects implantation. J Assist Reprod Genet 2007;24:111-7.

6. Shapiro BS, Daneshmand ST, Garner FC, et al. Evidence of impaired endometrial receptivity after ovarian stimulation for in vitro fertilization: a prospective randomized trial comparing fresh and frozen-thawed embryo transfer in normal responders. Fertil Steril 2011;96:344-8.

7. Blockeel C, Drakopoulos P, Santos-Ribeiro S, et al. A fresh look at the freeze-all protocol: a SWOT analysis. Hum Reprod 2016;31:491-7.

8. Roque M, Valle M, Guimaraes F, et al. Freeze-all policy: fresh vs. frozen-thawed embryo transfer. Fertil Steril 2015;103:1190-3.

9. Chen ZJ, Shi Y, Sun Y, et al. Fresh versus Frozen Embryos for Infertility in the Polycystic Ovary Syndrome. N Engl J Med 2016;375:523-33.

10. Shi Y, Sun Y, Hao C, et al. Transfer of Fresh versus Frozen Embryos in Ovulatory Women. N Engl J Med 2018;378:126-36.

11. Wei D, Liu JY, Sun Y, et al. Frozen versus fresh single blastocyst transfer in ovulatory women: a multicentre, randomised controlled trial. Lancet 2019;393:1310-8.

12. Alviggi C, Conforti A, Carbone IF, et al. Influence of cryopreservation on perinatal outcome after blastocystvs cleavage-stage embryo transfer: systematic review and meta-analysis. Ultrasound Obstet Gynecol 2018;51:54-63.

13. IVF-Worldwide Survey. Results: frozen-thawed embryo transfer. Available online: http://ivf-worldwide.com/ survey/frozen-thawed-embryo-transfer/results-frozenthawed-embryotransfer.html. Assessed June 23, 2019.

14. Volodarsky-Perel A, Eldar-Geva T, Holzer HE, et al. Cryopreserved embryo transfer: adjacent or non-adjacent to failed fresh long GnRH-agonist protocol IVF cycle. Reprod Biomed Online 2017;34:267-73.

15. Kaye L, Marsidi A, Rai P, et al. Frozen blastocyst transfer outcomes in immediate versus delayed subsequent cycles following GnRH agonist or hCG triggers. J Assist Reprod Genet 2018;35:669-75.

16. An Y, Sun Z, Li L, et al. Relationship between psychological stress and reproductive outcome in women undergoing in vitro fertilization treatment: psychological and neurohormonal assessment. J Assist Reprod Genet 2013;30:35-41.

17. Gameiro S, Boivin J, Domar A. Optimal in vitro fertilization in 2020 should reduce treatment burden and enhance care delivery for patients and staff. Fertil Steril 2013;100:302-9. 
18. Cesta CE, Viktorin A, Olsson H, et al. Depression, anxiety, and antidepressant treatment in women: association with in vitro fertilization outcome. Fertil Steril 2016;105:1594602.e3.

19. Santos-Ribeiro S, Polyzos NP, Lan VT, et al. The effect of an immediate frozen embryo transfer following a freeze-all protocol: a retrospective analysis from two centres. Hum Reprod 2016;31:2541-8.

20. Lattes K, Checa MA, Vassena R, et al. There is no evidence that the time from egg retrieval to embryo transfer affects live birth rates in a freeze-all strategy. Hum Reprod 2017;32:368-74.

21. Ozgur K, Bulut H, Berkkanoglu M, et al. Frozen embryo transfer can be performed in the cycle immediately following the freeze-all cycle. J Assist Reprod Genet 2018;35:135-42.

22. Bourdon M, Santulli P, Maignien C, et al. The interval between oocyte retrieval and frozen-thawed blastocyst transfer does not affect the live birth rate and obstetrical outcomes. PLoS One 2018;13:e0206067.

23. Kuang Y, Chen Q, Fu Y, et al. Medroxyprogesterone acetate is an effective oral alternative for preventing premature luteinizing hormone surges in women undergoing controlled ovarian hyperstimulation for in vitro fertilization. Fertil Steril 2015;104:62-70.e3.

24. Lu X, Hong Q, Sun L, et al. Dual trigger for final oocyte maturation improves the oocyte retrieval rate of suboptimal responders to gonadotropin-releasing hormone agonist. Fertil Steril 2016;106:1356-62.

25. Cummins JM, Breen TM, Harrison KL, et al. A formula for scoring human embryo growth rates in in vitro fertilization: its value in predicting pregnancy and in comparison with visual estimates of embryo quality. J In Vitro Fert Embryo Transf 1986;3:284-95.

26. Gardner DK, Schoolcraft WB. In vitro culture of human blastocyst. In: Jansen R, Mortimer D. editors. Towards

Cite this article as: Huang J, Lu X, Xie Q, Lin J, Cai R, Kuang Y. Timing of frozen-thawed embryo transfer after controlled ovarian stimulation in a non-elective freeze-all policy. Ann Transl Med 2019;7(23):752. doi: .21037/atm.2019.11.74 reproductive certainty: infertility and genetics beyond 1999. Carnforth: Parthenon Press, 1999:378-88.

27. Horowitz E, Mizrachi Y, Farhi J, et al. Modified naturalcycle cryopreserved embryo transfer: is a washout period needed after a failed fresh cycle? Reprod Biomed Online 2019;39:439-45.

28. Maas KH, Baker VL, Westphal LM, et al. Optimal timing of frozen embryo transfer after failed IVF attempt. Fertil Steril 2008;90:S285.

29. Santos-Ribeiro S, Siffain J, Polyzos NP, et al. To delay or not to delay a frozen embryo transfer after a failed fresh embryo transfer attempt? Fertil Steril 2016;105:1202-7.e1.

30. Higgins C, Healey M, Jatkar S, et al. Interval between IVF stimulation cycle and frozen embryo transfer: Is there a benefit to a delay between cycles? Aust N Z J Obstet Gynaecol 2018;58:217-21.

31. Casper RF. Introduction: Gonadotropin-releasing hormone agonist triggering of final follicular maturation for in vitro fertilization. Fertil Steril 2015;103:865-6.

32. Eldar-Geva T, Zylber-Haran E, Babayof R, et al. Similar outcome for cryopreserved embryo transfer following GnRH-antagonist/GnRH-agonist, GnRH-antagonist/ HCG or long protocol ovarian stimulation. Reprod Biomed Online 2007;14:148-54.

33. Huang R, Fang C, Xu S, et al. Premature progesterone rise negatively correlated with live birth rate in IVF cycles with GnRH agonist: an analysis of 2,566 cycles. Fertil Steril 2012;98:664-70.e2.

34. Conrad KP, Baker VL. Corpus luteal contribution to maternal pregnancy physiology and outcomes in assisted reproductive technologies. Am J Physiol Regul Integr Comp Physiol 2013;304:R69-72.

35. Chen X, Man GCW, Liu Y, et al. Physiological and pathological angiogenesis in endometrium at the time of embryo implantation. Am J Reprod Immunol 2017. doi: 10.1111/aji.12693. 


\section{Supplementary}

Table S1 Multivariable regression analysis for live birth before and after propensity score matching

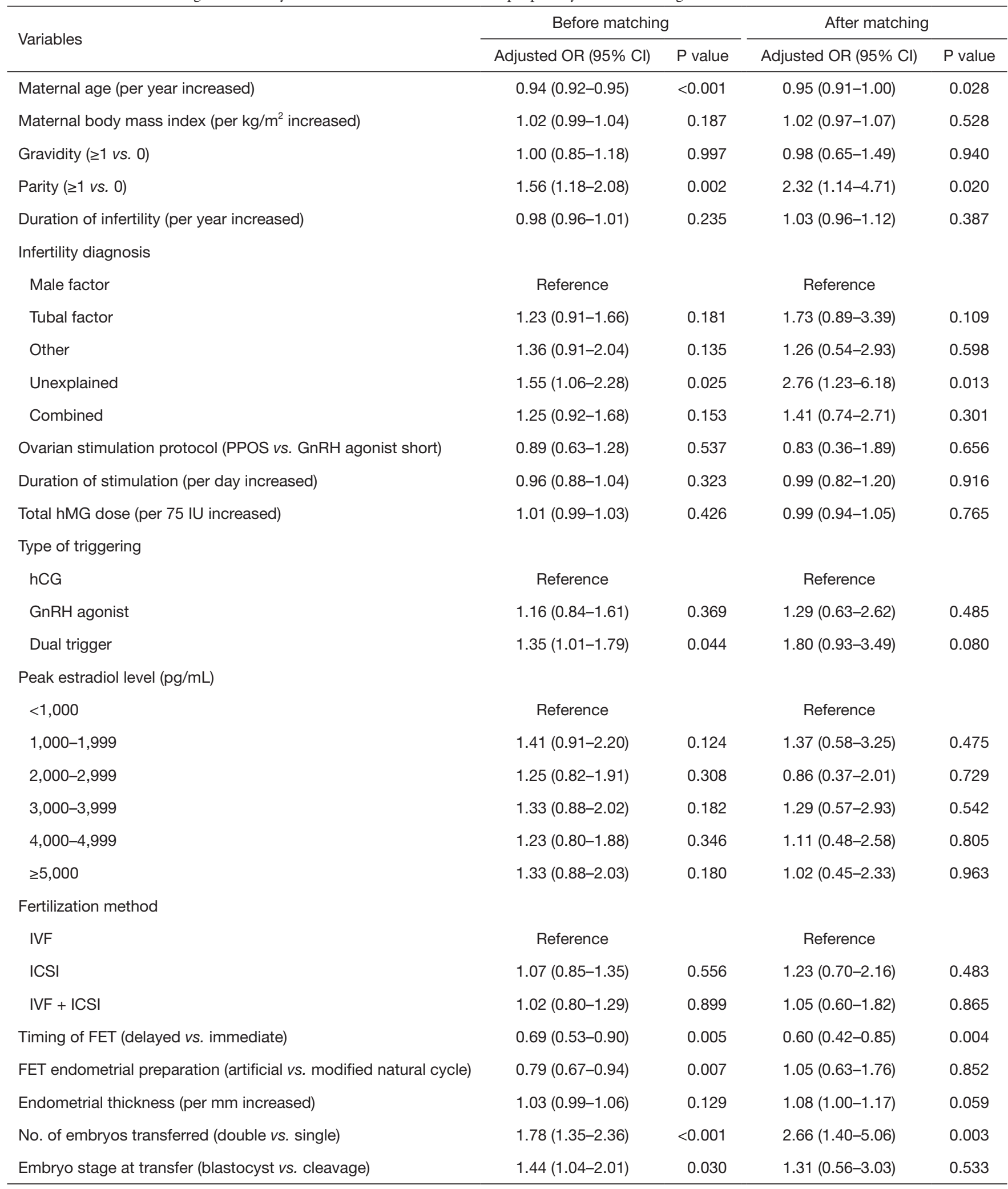

OR, odds ratio; Cl, confidence interval; PPOS, progestin-primed ovarian stimulation; GnRH, gonadotropin-releasing hormone; hMG, human menopausal gonadotropin; hCG, human chorionic gonadotropin; IVF, in vitro fertilization; ICSI, intracytoplasmic sperm injection; FET, frozen-thawed embryo transfer. 